\title{
La Prehistoria no está tan lejos de la actualidad: ciclo de mejora y metodología de aprendizaje en la asignatura de Prehistoria I
}

\section{Prehistory is not so far from today: improvement cycle and learning methodology in Prehistory I}

José A. Solano García

ORCID: https://orcid.org/0000-0001-6300-2351

Universidad de Sevilla

Departamento de Prehistoria y Arqueología

jgsolano@us.es

DOI: http://dx.doi.org/10.12795/9788447231003.066

Pp.: 1391-1411 


\section{Contexto de aprendizaje}

En el Doble grado de Geografía y Gestión del Territorio e Historia, de la universidad de Sevilla, se oferta la asignatura de Prehistoria I en el primer curso. En el curso 2020/2021 se han matriculado 30 alumnos. De los cuales, 26 han participado en la aplicación del CIMA y uno de ellos con necesidades académicas asociadas a discapacidad.

El CIMA se desarrolló entre el 11 y el 19 de noviembre, en cuatro clases de dos horas, bajo la modalidad de un escenario completamente online, dadas las circunstancias especiales de la pandemia COVID-19 y las restricciones impuestas por el gobierno. La docencia se ha impartido a través de la plataforma de enseñanza virtual (Blackboard) de la Universidad de Sevilla, que permite retransmitir las clases en vivo de manera síncrona y aportar contenido en la nube. Se trata de un escenario anormal que implica una merma en la relación humana directa y, por tanto, en la transmisión de la información (Porlán, 2020). Como veremos, se han integrado actividades colaborativas en el diseño del CIMA que favorecieran el acercamiento y la interacción entre los estudiantes y que aumentaran el grado de relación social.

Entre el temario de la asignatura de Prehistoria I se ha seleccionado, para la aplicación del CIMA, un tema sobre la organización económica y social de los Cazadores Recolectores (C-R) en el Pleistoceno. Esta temática ya fue abordada en cierta medida en la aplicación de otro CIMA en el año 2019 (Simón-Vallejo, 2019), sobre el origen del ser humano y su evolución. En cambio, en este trabajo nos centramos en aspectos relacionados con la economía, la sociedad y la ideología en su dimensión más antropológica, con una fuerte carga teórica y poco aplicada.

En contraste con el modelo de enseñanza-aprendizaje tradicional, basado en la "clase magistral" y en el profesor, se ha introducido un modelo de enseñanza centrado en el estudiante (Bain, 2005; Finkel, 2008; Porlán, 2017), 
implementando un modelo metodológico activo y constructivista. Su objetivo es aprender a aprender, en un doble sentido estudiante-profesor.

\section{Diseño previo del Ciclo de Mejora en el Aula}

Los objetivos del CIMA se centran en la calidad frente a la cantidad de contenidos, bajo un modelo de aprendizaje profundo. Donde los estudiantes irán construyendo progresivamente el conocimiento desde una fase básica inicial hasta una última que entraña mayor complejidad.

Entre los nueve objetivos generales que marca el Programa de la asignatura se han seleccionado los tres primeros como ejes estructurales del diseño y aplicación del ciclo. Además, se han establecido una serie de objetivos específicos en relación con el contenido.

\section{Objetivos generales}

- Ser capaz de interpretar y analizar las sociedades en su evolución espacio-temporal.

- Tener los conocimientos básicos de los procesos de investigación histórica.

- Saber transmitir los conocimientos y contenidos de la evolución histórica de las sociedades.

Objetivos específicos

a) Conceptuales:

- Aprender las características fundamentales y definitorias de los C-R.

- Establecer los límites geográficos y temporales en los que se desarrollan las comunidades de C-R en la Prehistoria antigua.

Ciclos de Mejora en el Aula (2020). Experiencias de Innovación Docente de la US Esta obra se distribuye con la licencia Creative Commons 
- Evolución, causas y consecuencias de las estrategias de subsistencia y modos de vida de los C-R a lo largo del Plio-pleistoceno.

b) Procedimentales:

- Analizar y sintetizar la estructura social de los cazadores recolectores.

- Aprender a realizar inferencias e hipótesis de rango medio sobre el pasado.

- Analizar y filtrar la información etnoarqueológica y experimental como complemento a la información del registro arqueológico.

c) Valores:

- Adquirir competencias clave de carácter social y cívico.

- Comparar las ideologías de igualitarismo social y laboral, de edad, de sexo y estatus con nuestra sociedad actual.

- Fomentar el respeto por la naturaleza y el medio ambiente y la sostenibilidad, como principal recurso de vida del ser humano.

- Valorar y respetar la vida espiritual, ritual y cultural en la diversidad social.

\section{Diseño de contenidos}

Sintetizar todo el conocimiento sobre el ser humano en su etapa más larga de la humanidad (más de dos millones de años), no es tarea fácil. La Prehistoria utiliza la Arqueología como herramienta metodológica científica y como su principal fuente de información. Conocemos que la Arqueología posee tres dimensiones: espacio, tiempo y forma; que se interrelacionan para generar el conocimiento histórico (Odriozola, 2019). Dado que el tiempo de ejecución del CIMA es reducido (8h), se ha optado por desprendernos, en gran medida, de dos de las dimensiones 
de la Arqueología: espacio y tiempo; para centrarnos en la forma. En el carácter universal del asunto en estudio: ¿Qué son? ¿Quiénes son? ¿Cómo viven los C-R? De manera atemporal y sin importar la localización geográfica puntual, pero sí la relación de estas comunidades con el medio natural o ecosistema en el que se desarrollan.

Al prescindir de la dimensión temporal podemos acercar el aprendizaje a la contemporaneidad, a la actualidad de los estudiantes. Podemos utilizar así los estudios antropológicos, etnográficos contemporáneos y la etnoarqueología para enseñar esta dimensión. Con la idea de reforzar en gran medida los canales de información en el proceso de enseñanza-aprendizaje.

Para acercar todavía más el contenido a los modelos mentales de los estudiantes, basados en su experiencia personal, se me ocurrió utilizar y adaptar la teoría de la motivación humana del psicólogo Abraham Maslow (1943), con un uso muy extendido en márquetin empresarial. En ésta se plantea un modelo jerárquico, en forma de pirámide, de las necesidades humanas, en el que la satisfacción de las necesidades más básicas da lugar a la necesidad de satisfacción de necesidades más altas. Si trasladamos este modelo a la evolución humana en la Prehistoria podríamos extrapolar esta progresión al propio proceso de evolutivo de las sociedades de los C-R. No se trata de una aplicación científica de investigación, donde quedarían muchos "flecos sueltos". Pero si puede cumplir su función en el proceso de aprendizaje, donde tenemos que descomponer en partes los contenidos del tema y establecer unos límites claros entre ellos para facilitar su asimilación.

A partir de esta propuesta, se han planteado cuatro grandes cuestiones o problemas clave, en relación con las necesidades propias del ser humano y que engloban todo el contenido sobre la organización económica y social de los C-R (Figura 1):

Ciclos de Mejora en el Aula (2020). Experiencias de Innovación Docente de la US Esta obra se distribuye con la licencia Creative Commons 
1. ¿Cuáles son las necesidades básicas (físicas) de estas sociedades y cómo las cubren?

2. ¿Qué estrategias de subsistencia utilizan para cubrir sus necesidades de refugio y seguridad?

3. ¿Qué estrategias y estructuras sociales desarrollan para mantener sus modos de vida?

4. ¿Cuál es su ideología y qué fórmulas adoptan para explicar los fenómenos de la naturaleza; entre ellos la vida y la muerte?

Paleolítico superior

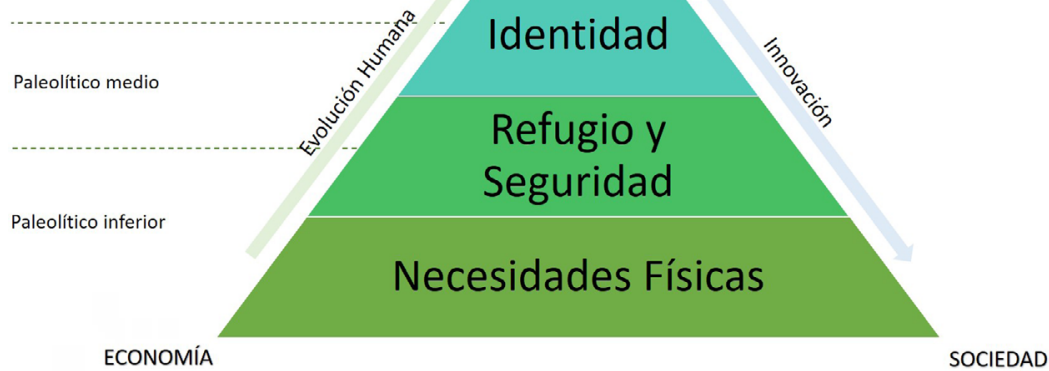

Figura 1. Esquema de problemas clave estructurados jerárquicamente que sintetizan la evolución humana de las organización económica, social y simbólica de las comunidades de C-R en la Prehistoria.

Los contenidos se han repartido y agrupado en cada uno de sus bloques correspondientes mencionados (Figura 2), más otros dos bloques generales que inciden en todo el contenido: los contenidos relacionados con la definición de estas sociedades y la metodología de investigación. Estos últimos son tratados colateral o indirectamente durante el desarrollo de los bloques principales.

Ciclos de Mejora en el Aula (2020). Experiencias de Innovación Docente de la US Esta obra se distribuye con la licencia Creative Commons 


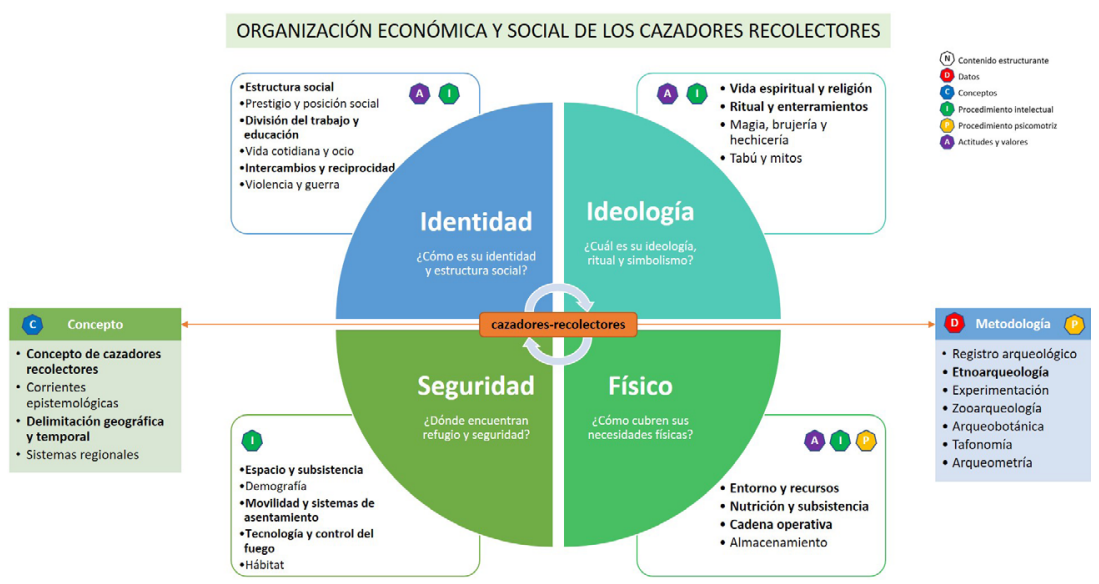

Figura 2. Propuesta de contenidos. Alrededor de los cuatro problemas clave, se estructuran todos los contenidos y se interrelacionan.

Puesto que uno de los principios metodológicos del CIMA consiste en formular contenidos de aprendizaje de diversos tipos para promover una formación integral de los estudiantes, se han seleccionado dentro de cada bloque aquellos que por carácter estructurante e importancia mantienen la estructura relacional del conjunto. Además, dentro de éstos, se ha buscado la diversidad a través de contenidos conceptuales, procedimentales y actitudinales.

\section{Modelo metodológico}

El modelo metodológico está basado en el principio de investigación activo y constructivista (Figura 3). Este modelo responde a cada uno de los núcleos centrales o grandes cuestiones en las que se ha sintetizado el tema. De tal manera, que cada gran pregunta constituye un bloque de trabajo para cada una de las cuatro sesiones.

La inmersión de los alumnos comenzará con plantear la complejidad y dificultad de comprender la organización económica y social de los C-R en el escenario de 
investigación prehistórica formulando las cuatro grandes cuestiones generales que sintetizan los aspectos fundamentales del tema ya mencionadas. A la vez que se les comenta las distintas herramientas metodológicas de investigación arqueológica con las que se abordan.

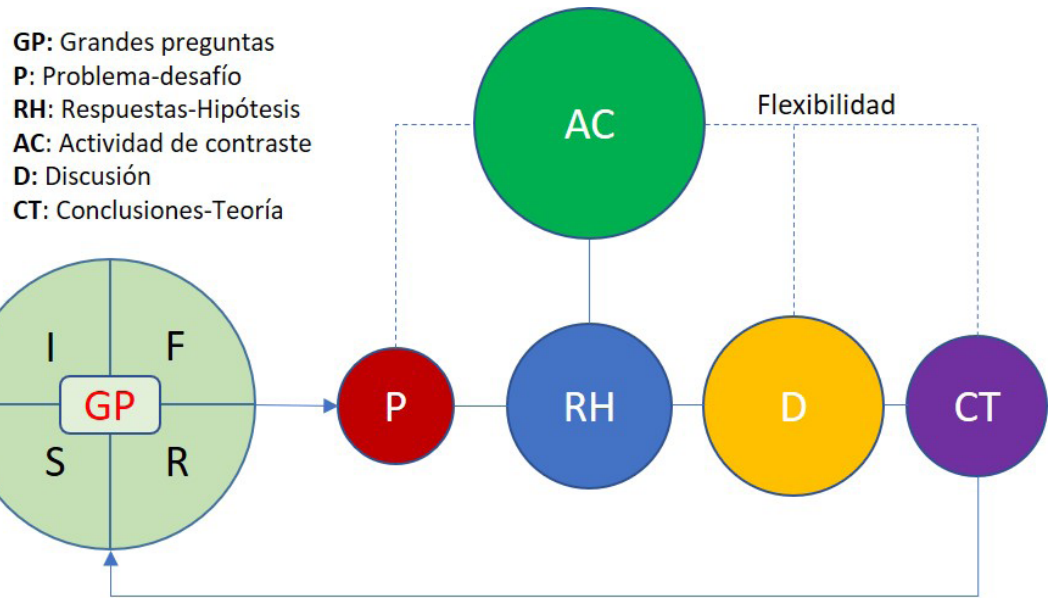

Figura 3. Modelo metodológico posible implementado en el CIMA.

Partimos del planteamiento de un problema o desafio $(P)$, relacionado con el contenido de los problemas clave o núcleo centrales (GP), al que se tienen que enfrentar los alumnos y cuya función es activar rápidamente su mente e imaginación, para lanzar ideas e hipótesis ( $\mathrm{RH}$ ) que tendrán que contrastar (AC). La actividad de contraste tiene como finalidad que los estudiantes comiencen a reelaborar sus ideas. Una vez realizada, expondrán sus resultados y se debatirá sobre ellos (D). Deberán defender su postura a partir de la actividad de contraste realizada y la experiencia, de manera individual o en grupo según se haya diseñado dicha actividad. Una vez discutidos los resultados se buscará que acuerden unas conclusiones (CT) como respuestas al problema-desafío planteado y, con la ayuda del profesor, decidir cómo podemos insertarlo en un modelo general que responda a las grandes cuestiones.

Ciclos de Mejora en el Aula (2020). Experiencias de Innovación Docente de la US Esta obra se distribuye con la licencia Creative Commons 
Se trata de un modelo metodológico sencillo que busca la reflexión del alumno y la interacción y la capacidad de interconectar ideas más que consumir datos. Esa reflexión está presente en todos los pasos del modelo, de manera que la actividad de contraste le provoca no ya solo respuestas al pequeño problema-desafío, sino nuevas preguntas a reformular, nuevas ideas que discutir y posibles soluciones a un corpus mayor abierto a distintas interpretaciones.

La resolución de pequeños problemas, asociados a los contenidos seleccionados, son como piezas de una estructura que los estudiantes ensamblan, construyendo poco a poco el andamiaje del aprendizaje (Porlán, 2017). En esta construcción prima la flexibilidad marcada por la retroalimentación del modelo, pudiendo andar y desandar para reconstruir el conocimiento. En cualquiera de las fases se puede volver a una idea original, a la actividad de contraste o al punto de partida para reformular otra pregunta.

\section{Secuencia de actividades}

El cronograma y la secuencia de actividades se ha diseñado y programado acorde al modelo metodológico propuesto y al diseño de contenidos. En principio, cada uno de los cuatro núcleos centrales se encuadra en una de las cuatro sesiones. Para cada una se ha diseñado una hoja de ruta, con el contenido de cada sesión, que sirve de guía para profesor y alumno. En ella pueden seguir todos los pasos programados en la secuencia de actividades, junto con los recursos necesarios o enlaces a ellos para realizar las actividades de contraste.

En la elaboración y diseño de la secuencia de actividades se ha dado mucho valor a la formación de grupos de trabajo y a la interacción para la resolución de problemas, 
en contraste con la importancia de comprender las relaciones e interacciones intra e intergrupales de los cazadores recolectores como medo de cohesión en sus estrategias de subsistencia para resolver problemas de supervivencia.

Tabla 1. Ejemplo secuencia de actividades del CIMA.

\begin{tabular}{|c|c|c|c|}
\hline ACTIVIDAD & DESCRIPCIÓN & FASE & TIEMPO \\
\hline 1 & Problema-desafío & $P$ & $15^{\prime}$ \\
\hline \multicolumn{4}{|c|}{$\begin{array}{l}\text { ¿Qué es la identidad? ¿Qué es el simbolismo? ¿Cómo codifican la información } \\
\text { los C-R? ¿Podemos interpretar su pensamiento a través del registro } \\
\text { arqueológico? }\end{array}$} \\
\hline 2 & Primeras ideas e hipótesis & $\overline{\mathrm{RH}}$ & $15^{\prime}$ \\
\hline \multicolumn{4}{|c|}{$\begin{array}{l}\text { Los alumnos apuntarán con frases cortas o palabras sueltas las ideas que } \\
\text { le vayan surgiendo sobre el problema propuesto. A la vez que pasarán a } \\
\text { expresarlas a toda la clase. Se les animará a que formulen hipótesis que } \\
\text { puedan dar respuesta al problema planteado y que las anoten en su hoja de } \\
\text { ruta, antes de pasar a la actividad de contraste. }\end{array}$} \\
\hline 3 & Actividad de contraste & $A C$ & $15^{\prime}$ \\
\hline \multicolumn{4}{|c|}{$\begin{array}{l}\text { Juego de rol sobre la identidad grupal, el simbolismo y la transmisión de la } \\
\text { información. A cada grupo se le asigna un ecosistema concreto de hábitat. } \\
\text { El grupo tendrá que definir su economía y estructura social a partir de su } \\
\text { relación con el entorno natural. Una vez definido, tendrán que codificar y } \\
\text { diseñar un símbolo de identidad que refleje las características del grupo. } \\
\text { Cada grupo expondrá su simbolo y el resto de grupos intentará interpretar } \\
\text { su significado, de tal manera que conozcamos los aspectos básicos de } \\
\text { organización de ese grupo. }\end{array}$} \\
\hline 4 & Exposición de resultados y discusión & $\mathrm{RH}$ & $15^{\prime}$ \\
\hline \multicolumn{4}{|c|}{$\begin{array}{l}\text { Cada grupo mostrará su simbolo/s al resto de grupos, que tendrán que } \\
\text { interpretarlo para conocer la identidad y organización de éste. ¿Qué } \\
\text { obstáculos encontramos? } \\
\text { Se les animará a que formulen hipótesis que puedan dar respuesta a las } \\
\text { cuestiones del problema-juego planteado. }\end{array}$} \\
\hline 5 & Contraste de ideas e hipótesis & $\overline{A C}$ & $30^{\prime}$ \\
\hline \multicolumn{4}{|c|}{$\begin{array}{l}\text { Lectura de una investigación etnoarqueológica (Hernando, 2001) sobre } \\
\text { identidad. } \\
\text { Visionado de un video documental con especial mención a la ideología. }\end{array}$} \\
\hline 6 & Exposición de resultados y discusión & & $15^{\prime}$ \\
\hline
\end{tabular}

Ciclos de Mejora en el Aula (2020). Experiencias de Innovación Docente de la US Esta obra se distribuye con la licencia Creative Commons Reconocimiento-NoComercial-SinObraDerivada Internacional (CC BY-NC-ND 4.0.) 
Se establecerá un debate final sobre la formación de la identidad, el simbolismo y la transmisión de la información, la espiritualidad y la cultura de la muerte en la Prehistoria.

\section{7} Sintesis y formulación de un modelo

CT $15^{\prime}$

A partir de la actividad de contraste y la reelaboración de ideas del debate, junto con una pequeña píldora de información sobre las distintas maneras de entender el mundo y el más allá y de expresarlo, se concluirá con los distintos modelos de aproximación para interpretar la ideología, a pesar de su dificultad.

\section{Aplicación del Ciclo de Mejora en el Aula}

En la sesión uno se presentó el contenido auspiciado bajo los cuatro bloques estructuradores y la metodología de aprendizaje que íbamos a seguir. A continuación, se realizó un ejercicio de inmersión de los alumnos en las sociedades cazadoras-recolectoras contemporáneas. Como recurso se utilizaron dos videos, tipo documental; por su marcado carácter cinematográfico y, por tanto, más inmersivos: 1) sobre el paisaje y ecosistemas de la sabana africana y 2) otro donde introducimos a un grupo de bosquimanos en interacción con ese medio natural.

A partir de ahí se fueron desarrollando el resto de las actividades según el modelo metodológico diseñado. Todo de manera muy dinámica; lecturas rápidas sin detenimiento para apoderarse de conceptos, discutirlos y reelaborar sus ideas antes de pasar al siguiente problema. Al finalizar cada sesión acordamos entre todos unas conclusiones en forma de modelo teórico que pudieran dar respuesta a la cuestión principal del bloque en estudio.

El carácter humano de este tema los acerca mucho a su experiencia personal y a dar opiniones. Sin embargo, el desarrollo completamente online del aprendizaje ocultaba en gran medida el seguimiento del contenido por una parte del alumnado que no participaba. Entre la sesión dos y la sesión tres, se propusieron dos actividades a 
desarrollar fuera del aula, durante el fin de semana: 1) visionado y análisis crítico de una película que transmite las consecuencias de desequilibrio que pueden surgir en la interacción de una sociedad moderna occidental con una de C-R; y 2) un juego de rol en el que a partir de 6 grupos creados de forma aleatoria debían de buscar la mejor configuración del grupo en cuanto a género, a partir de la interacción e intercambio de miembros con el resto de grupos, para asegurar el equilibrio económico y social a la vez que su supervivencia, ante una probable extinción.

En el análisis y debate de la película, aunque no les gustó mucho, por su marcado desfase temporal, sí que captaron el mensaje y sobre todo observaron y aprehendieron las diferencias económicas y sociales entre el mundo occidental actual y una sociedad anclada en economías de subsistencia. Cambió su manera de observar a estas sociedades.

El juego de rol aumentó la participación de los alumnos. Cada grupo eligió un representante para exponer su estrategia de supervivencia a partir de la selección de género, motivados por una recompensa para el grupo que diseñara la estrategia óptima y la argumentase. Alguno de los grupos incluso recreó todo un escenario geográfico, económico y social contextualizado en la actualidad. Increíble la capacidad de imaginación cuando se sienten motivados. A pesar de ello, un grupo se quedó rezagado por la falta de cohesión e interacción entre sus miembros. Esta actividad, les resultó muy motivadora. Habían puesto en práctica y en marcha sin darse cuenta justo el contenido del bloque en activo, sobre las relaciones sociales en las bandas de C-R. Ellos eran los protagonistas.

Ahora tan solo tenían que tomar los conceptos clave a partir de las actividades de contraste para reelaborar sus ideas y componer la estructura social. Además, el contenido 
estructurado de este bloque sobre la división del trabajo y diferenciación de género y edad, de gran actualidad, hizo fluir la sesión a partir del ritmo marcado por ellos. El cronograma de actividades pasó a ser algo secundario. Podría ser inquietante, pero armonicé los tiempos ad hoc para alargar los debates y reducir las lecturas e incluso el visionado del documental-inmersivo (ya no era necesario). Una vez alcanzado este clímax, creí que era conveniente no romperlo al menos durante esta sesión.

En la última sesión, sobre ideología, comenzamos a trabajar sobre el concepto de identidad. Se planteó a los alumnos un nuevo juego a partir de los grupos anteriores ya creados, en el que a cada grupo debía recrear una banda de C-R a partir de un ecosistema asignado y codificar sus características en un símbolo. El resto de los grupos intentaría a partir del símbolo analizar y conocer la identidad de los demás. Esta actividad fue altamente participativa y excedió los límites de tiempo. Los alumnos estaban experimentando de "primera mano" la realidad de las sociedades de C-R. Tras la actividad de contraste y discusión se elaboraron las conclusiones finales. Ahora estaban en disposición de relacionar los cuatro grandes bloques para construir como pudieron ser estas sociedades en el pasado. En la última media hora realizaron de nuevo el mismo cuestionario.

\section{Análisis y evaluación del Ciclo de Mejora en el Aula}

La evaluación del ciclo está en estrecha relación con su éxito en la progresión del alumno, en que el mayor número de ellos progrese. Para ello es necesario explorar los modelos de los estudiantes, a partir de su expresión oral, escrita o artística (Porlán, 2017). La realización de un cuestionario inicial y final nos permite en cierta medida

Ciclos de Mejora en el Aula (2020). Experiencias de Innovación Docente de la US Esta obra se distribuye con la licencia Creative Commons 
acceder a esa información y medir e inferir dos cuestiones básicas: el progreso de los estudiantes y el grado de éxito de nuestro ciclo de mejora.

\section{Análisis del cuestionario de aprendizaje}

En la elaboración del cuestionario se ha tenido en cuenta el diseño del mapa de contenido para tratar los problemas clave y una formulación de las preguntas adecuada y cercana a su experiencia que les permitiera expresarse con libertad (Rivero et al., 2017). Se confeccionó a partir de diez preguntas de respuesta libre. Cuatro preguntas sobre los cuatro problemas clave o bloques de contenido y otras cuatro complementarias que profundizaban y preguntaban de forma más directa sobre asuntos estructurantes de cada uno de los bloques. Las dos restantes son de tipo exploratorio: 1) ¿Qué crees que son los cazadores-recolectores? 2) ¿En crees que se basa la economía de los cazadores-recolectores? 3) ¿En qué periodos de la historia de la humanidad piensas que se encuentran las sociedades de cazadores-recolectores? 4) ¿Sabrías indicar que estrategias de subsistencia utilizan los cazadores-recolectores para cubrir sus necesidades básicas? 5) ¿Y para cubrir sus necesidades de protección y seguridad en el mundo que les rodea? 6) ¿Crees que las sociedades de cazadores-recolectores están compuestas por grandes poblaciones? ¿Permanecen siempre en el mismo lugar? 7) ¿Cómo crees que son las relaciones sociales entre los cazadores-recolectores? ¿Las relaciones entre ellos, entre otros grupos? 8) ¿En que suelen trabajar los cazadores-recolectores y cómo piensas que se reparten el trabajo? ¿Crees qué tendrán vacaciones? 9) ¿Cómo crees que los cazadores-recolectores ven el mundo que les rodea, el sentido de la vida, el sentido de la muerte, la religión o en qué crees que piensan? 10) ¿Qué opinión te merecen las sociedades de cazadores recolectores? Ciclos de Mejora en el Aula (2020). Experiencias de Innovación Docente de la US
Esta obra se distribuye con la licencia Creative Commons 
Se han categorizado las respuestas en cinco grandes bloques en gradación de complejidad; desde el más inferior (E), cuando no se contesta a la pregunta, al superior (A), que comporta una aproximación compleja de interrelación de todo el contenido. Además, se ha cuantificado cada una de las respuestas en función del grado de complejidad para realizar diversos análisis cuantitativos.

Al realizar un primer análisis exploratorio (Figura 4) se observa el grado de variabilidad en las respuestas de los estudiantes, sobre todo en las preguntas de opinión. En cambio, existen una mayor homogeneidad en las preguntas directas de profundización complementaria, donde la gran mayoría de estudiantes contestan de manera similar.

En cuanto al nivel de conocimiento y concreción en sus respuestas, la mayoría se encuentra en el nivel $C$, seguido del B. Las preguntas 1-3 y 8, con mayoría en el nivel C, abordan aspectos básicos que necesariamente tienen que ser corregidos para abordar contenidos más complejos.
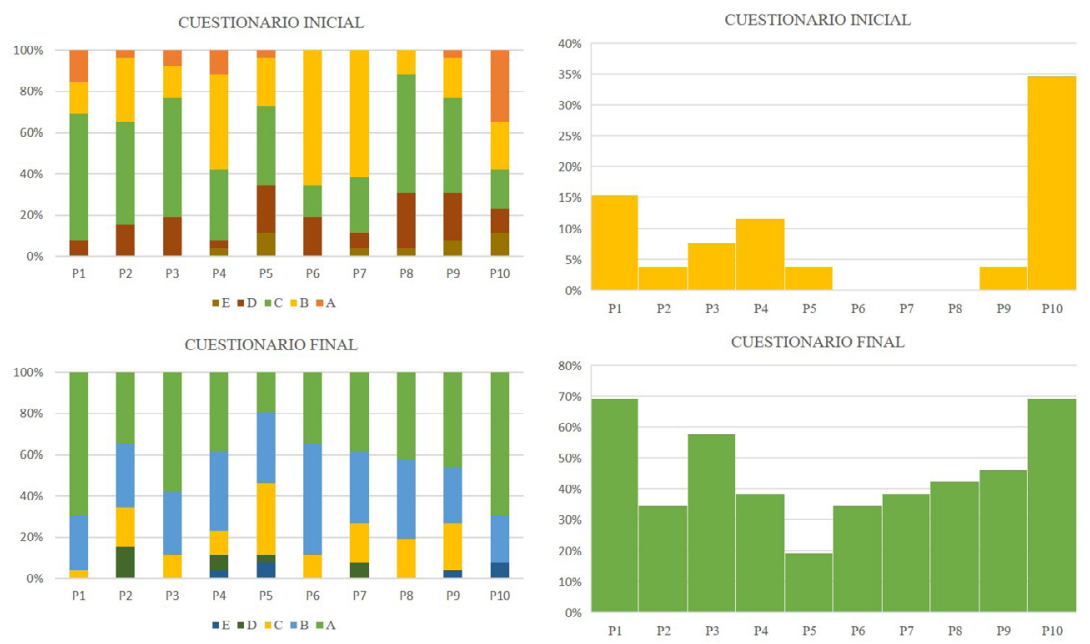

Figura 4. Comparativa del cuestionario inicia y final. Gráfico de barras apiladas (izquierda) con la proporción de respuestas por categoría de cada pregunta. Histograma de frecuencias (derecha) de categorías por pregunta. Ciclos de Mejora en el Aula (2020). Experiencias de Innovación Docente de la US
Esta obra se distribuye con la licencia Creative Commons 
A partir del escenario descrito se plantean dos objetivos: corregir la variabilidad de respuestas hacia la normalización e intentar trasladar a los alumnos a categorías superiores de aprendizaje. Sobre todo, reducir las categorías de aprendizaje D y C. Al analizar el cuestionario final (Figura 4) observamos ya en el histograma una mayor homogeneidad en los resultados. Se ha equilibrado en gran medida los modelos mentales de los estudiantes. Sobre todo, se refleja en las preguntas 6,7 y 8 , que entrañan cierta complejidad y ahora se han normalizado.

En cuanto al segundo objetivo, podemos observar que se han reducido en una gran proporción las respuestas asociadas a las categorías D y C y se ha producido una mayor concentración en las categorías A y B de todas las preguntas. Se ha realizado un notable progreso en el modelo de aprendizaje.

\section{Análisis de las escaleras de aprendizaje}

Se han realizado dos "escaleras de aprendizaje" que reflejan la progresión del estudiante (Porlán, 2017: 81). A partir de la matriz de datos cuantitativos por cada respuesta de cada alumno a todas las preguntas, se han sintetizado en una escalera inicial y otra final (Figura 5).

Antes de la aplicación del ciclo de mejora, el $41 \%$ de los estudiantes adoptaba una postura determinista y funcionalista de las bandas de C-R, aunque con un 31\% que establecía algunas relaciones básicas de economía y sociedad. Aunqu un 8\% alcanzaba un nivel de compresión mayor en la interrelación del contenido, existía una preocupante proporción de alumnos (16\%) con gran confusión conceptual e, incluso, un $5 \%$ que no se atrevía a ofrecer una respuesta.

Ciclos de Mejora en el Aula (2020). Experiencias de Innovación Docente de la US Esta obra se distribuye con la licencia Creative Commons 
En contraste, como resultado final de la aplicación del ciclo de mejora, el $20 \%$ de los dos niveles más bajos ha disminuido al 5\%. Una mejora muy importante para los alumnos de nivel más bajo. El resto de la escalera final muestra un paulatino progreso hacía los niveles más altos de aprendizaje. El nivel C inicial (41\%), era prácticamente el punto de partida de la mayoría de alumnos. Ahora se han repartido entre las categorías $B$ y $A$, siendo ésta última (45\%) la que han alcanzado la mayoría de los estudiantes.

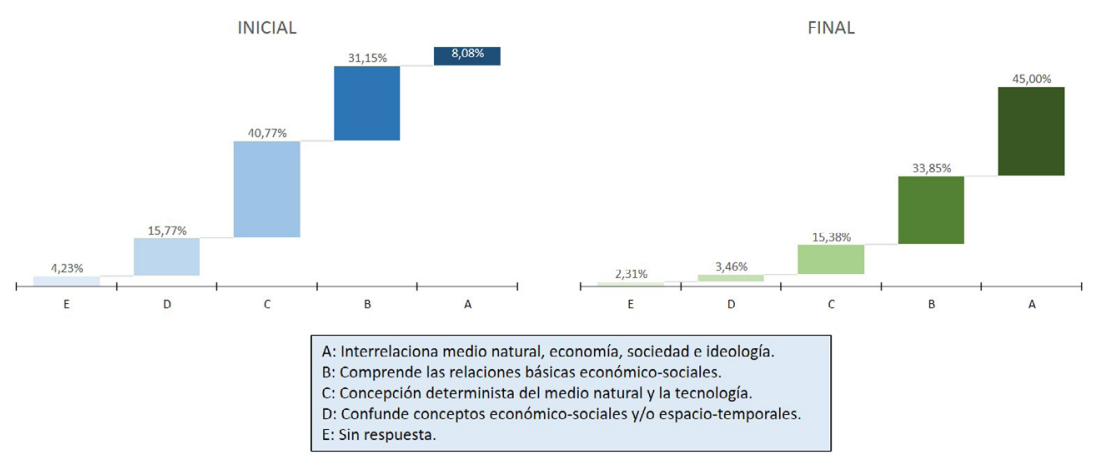

Figura 5. Comparativa entre la escalera de aprendizaje inicial y final.

Pudiera parecer que el resultado óptimo fuese una gran concentración de estudiantes en el nivel más alto, por encima del $75 \%$, pero creo que el resultado responde a una progresión lógica del aprendizaje. No se puede alcanzar el nivel superior sin rebasar cada uno de los obstáculos anteriores. La tendencia de la escalera final es coherente con esta lógica.

\section{Análisis del aprendizaje de los estudiantes}

En general, se ha producido un notable progreso en el aprendizaje de los estudiantes (Figura 6), del 58\% al 83\%. La media es de 23 puntos por encima del momento inicial, aunque con una desviación estándar del 17,4\%. A pesar de haber conseguido una homogenización del aprendizaje, hay cierta variabilidad por los dos extremos: alumnos que

Ciclos de Mejora en el Aula (2020). Experiencias de Innovación Docente de la US Esta obra se distribuye con la licencia Creative Commons 
han despuntado con un progreso muy acelerado y estudiantes que no es que no hayan mejorado, sino que han empeorado. La primera conclusión no es tan preocupante como la segunda. ¿Qué ha ocurrido con estos estudiantes? ¿Por qué su nivel de aprendizaje se ha visto mermado? Para contestar a estas cuestiones no basta con la lectura y análisis de los datos que presentamos, sino que sería necesario realizar una entrevista con dichos alumnos.
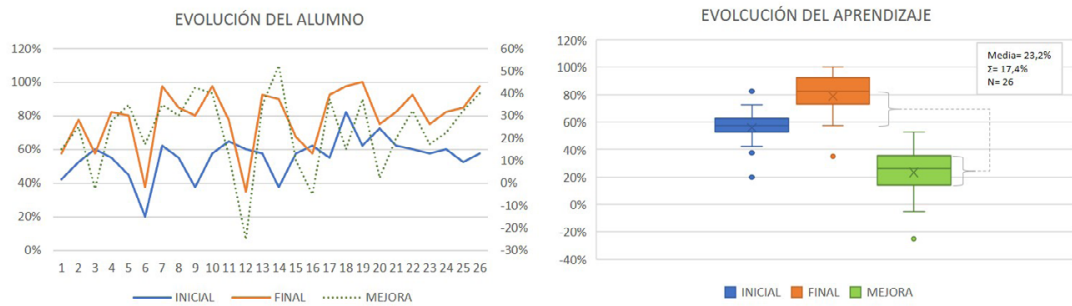

Figura 6. Evolución del progreso de los estudiantes en la aplicación del ciclo de mejora.

Los alumnos 3, 12, 16 y 20 han visto sufrido una caída en su rendimiento durante la aplicación del ciclo de mejora. Si analizamos la tabla cruzada de respuestas de los alumnos podemos observar una caída del aprendizaje en cuestiones concretas. El alumno 3 ha descendido en preguntas centrales sobre aspectos básicos de economía de estas sociedades que tal vez no ha alcanzado a comprender. Mientras que los alumnos 12 y 16 lo han hecho a en preguntas de mayor complejidad sobre aspectos relacionados con la sociedad y la ideología.

Resulta extraño este escenario de descenso cuando el resto de los alumnos ha conseguido una notable mejora. El hecho de haber realizado la docencia completamente online podría ser la raíz del problema, aunque dentro de éste, los escenarios pueden ser múltiples; pérdida de interés, dejadez y desidia, fallos múltiples de conexión, etc. Como decía, una entrevista personal podría aclarar en gran medida esta cuestión, pendiente de realizar.

Ciclos de Mejora en el Aula (2020). Experiencias de Innovación Docente de la US Esta obra se distribuye con la licencia Creative Commons 


\section{Consideraciones finales y principios didácticos}

La introducción de este ciclo de mejora ha resultado muy satisfactoria, tanto a nivel docente como discente. Existía un gran riesgo de compromiso por parte de los alumnos al realizar todo el aprendizaje online, sin embargo, la gran mayoría ha adoptado un papel activo, demostrando su implicación, ganas por aprender y de madurez insospechada.

Los resultados positivos en la evolución de aprendizaje se han puesto de manifiesto en el análisis y evaluación del ciclo. Una gran proporción de alumnos ha visto incrementado su aprendizaje sobre los contenidos estructurantes a un nivel universal. Esto es, cercano al mundo que les rodea, donde comprendan mejor la realidad social de las comunidades "indígenas" actuales, su valor inmaterial como patrimonio de la humanidad, que refleja la base de nuestros orígenes en la evolución humana. Se ha huido de la enseñanza tradicional hacia un modelo que fomente la libertad, la actitud crítica, la solidaridad, la sostenibilidad y el trabajo en equipo; valores y capacidad de reflexión para su vida postuniversitaria.

Un aspecto que me ha parecido clave durante la aplicación del ciclo ha sido el de la flexibilidad en el seguimiento de la hoja de ruta de actividades. Pienso que es básico disponer de ella como elemento vertebrador del proceso de aprendizaje, tanto para el docente como para los alumnos. Algunas actividades han resultado muy estimulantes para ellos y para mí; aquellas relacionadas con la interacción activa y social de los grupos, estimulando el ingenio, la audacia y la perspicacia en las propuestas a resolver y, generando divertidos y edificantes debates. Es por ello, que no podemos encorsetarnos en un guion rígido preestablecido, sino que debe primar la flexibilidad y contener, tal vez, alternativas gancho que nos sirvan para escalar o descender dentro de los eslabones del guion. 


\section{Principios didácticos}

- Acercar los contenidos a la realidad económica y social del alumnado produce mayor seguridad en ellos, entienden mejor los contenidos y conceptos, a la vez que ven su posible aplicación en el mundo actual; personal y laboral.

- Plantear los problemas clave como algo que les concierne a ellos, a pesar de tratarse de una realidad de hace más de diez mil años. Las necesidades de las sociedades pasadas son al final las mismas que tenemos nosotros hoy en día.

- Frente al uso de nuevos recursos tecnológicos se ha fomentado muchísimo la interrelación social entre ellos, con un fuerte grado de inmersión en los problemas clave, lo que ha generado una alta participación, pero sobre todo un deseo explícito de avidez de conocimiento y, por tanto, de investigación. Se ha despertado la curiosidad.

- Obviar en algunas ocasiones los tecnicismos y conceptos epistemológicos científicos intelectuales y emprender una vía del conocimiento cercana a ellos, a su experiencia, pero sin desvirtuar el contenido científico, ofrece la seguridad necesaria para la asimilación de conceptos complejos y los afianza.

- Intentar soslayar el sistema de evaluación tradicional hacia un modelo de evaluación continua alumno-profesor. El uso de un diario tal vez sea la mejor herramienta (Porlán y Martín, 1991), en un proceso día a día de observación del aprendizaje.

Ciclos de Mejora en el Aula (2020). Experiencias de Innovación Docente de la US Esta obra se distribuye con la licencia Creative Commons 
Palabras clave: Doble grado en Geografía y Gestión del Territorio e Historia, Prehistoria I, Cazadores-recolectores, Docencia universitaria, Experimentación docente universitaria.

Keywords: Double degree in Geography and Land Management and History, Prehistory I, Hunter-gatherers, University teaching, University Teaching Experimentation.

\section{Referencias bibliográficas}

Bain, K. (2005). Lo que hacen los mejores profesores universitarios. Valencia: Publicaciones Universidad de Valencia.

Finkel, D. (2008). Dar clases con la boca cerrada. Valencia: Publicaciones Universidad de Valencia.

Maslow, A.H. (1943). A theory of human motivation. Psychological Review, 50(4), 370-396.

Odriozola, C. (2019). Un ciclo de mejora en el aula aplicado en la asignatura de Análisis de Artefactos. En R. Porlán y E. Navarro (Coords.), Ciclos de mejora en el aula año 2019. Experiencias de innovación docente de la Universidad de Sevilla (pp. 424-448). Sevilla: Editorial Universidad de Sevilla.

Porlán, R. Coord. (2017). Enseñanza universitaria. Cómo mejorarla. Madrid: Morata.

Porlán, R. (2020). El cambio de la enseñanza y el aprendizaje en tiempos de pandemia. Revista de Educación Ambiental y Sostenibilidad 2(1), 1502.

Porlán, R. y Martín, J. (1991). El diario del profesor. Un recurso para la investigación en el aula. Sevilla: Díada.

Simón-Vallejo, M.D. (2019). Ciclo de Mejora Docente en el Aula aplicado a la asignatura de Prehistoria I: Las Sociedades Cazadoras y Recolectoras. Grado en Historia (Universidad de Sevilla). En R. Porlán y E. Navarro (Coords.), Ciclos de mejora en el aula año 2019. Experiencias de innovación docente de la Universidad de Sevilla (pp. 2613-2630). Sevilla: Editorial Universidad de Sevilla.

Ciclos de Mejora en el Aula (2020). Experiencias de Innovación Docente de la US Esta obra se distribuye con la licencia Creative Commons 